\title{
Informant-Made Videos: A Research and
}

\section{Educational Tool}

\author{
Richard Lachapelle 1 \\ Concordia University, Montreal
}

\begin{abstract}
1This paper is based on
the doctoral dissertation

Aesthetic Understanding as

Informed Experience: Ten

Informant-Made

Videographic Accounts

about the Process of

Aesthetic Learning (1994),
\end{abstract}

Concordia University,

Montreal, Quebec. The

author wishes to express

his gratitude to the Getty

Center for Education in

the Arts (Doctoral

Dissertation Fellowship

Program) for its financial

support of this study.

The author also wishes to

acknowledge the consid-

erable and valuable con-

tribution of his disserta-

tion advisor, David

Pariser, to this research

effort. Correspondence

concerning this article

should be addressed to

the author at:

Department of Art

Education, VA-208,

Concordia University,

1455 de Maisonneuve

Boulevard West,

Montreal, Quebec H3G

1M8, Canada.

\begin{abstract}
This study documents the use of informant-made video (IMV) recordings as a data collecting method for research into adults' art viewing experiences. Using discourse analysis, ten informants' own videotaped accounts of their responses to works of art were compared to audiotaped baseline responses collected using a traditional interviewing method. The cinematographic orientation and non-narrated segments of the IMVs were also analyzed. The IMVs were shown to be equal to the audiotaped interview as a means of collecting verbal statements about the works of art. Furthermore, the IMVs were found to provide additional information including visual documentation of the artworks, the tracking of informants' trajectories, and documentation of informants' gestures and physical interaction with the works of art. The author concludes with a discussion of the numerous educational possibilities of IMVs.
\end{abstract}

The art viewing experience is a complex one that defies simple description. A response to a work of art tends to unfold quickly and, as it proceeds, it tends to take on ever-shifting forms. It may begin as an emotional response and then, minutes later, transform itself into an intellectual or a sensate response. The researcher studying art viewing experiences must often work in an extremely uncontrolled and ever-changing environment. Under such conditions, most observers' skills are taxed to the limit. From the point of view of the researcher, the tool discussed in this article-the informant-made video-has the potential to lessen that strain by permitting investigators to review events as often as is necessary to fully understand them.

In the following excerpt from a post-study interview, a study informant conveys her point of view about the experience of using a video camcorder to make a record of her responses to two different works of art.

I felt that my words would be understood because the visual component would be there to back up what I was saying....I felt that the video would pick up a lot of what I was trying to express such as...how I was actually physically or visually looking at a work....I was hoping that the camera would somehow show... how I first took in the whole image; how I would then come in closer; how I would then take in details; how my eye moved around. (Lachapelle, 1993, p. 335)

This paper presents the findings of a research project conducted in part to explore the feasibility of using informant-made video recordings as a source of raw data for research into adults' art viewing experiences. Under 
the guidance of a researcher, informant-made videos(IMV) are video recordings produced by study participants in order to document their own art viewing experiences; informants make these recordings using portable video cameras, more commonly known as "camcorders."

Informant-made videos provide a number of advantages.

(1) As pointed out by Heider (1976, p. 43), IMVs reduce the possibility that the data collected for the purposes of research will be influenced by a cultural or other bias on the part of the researcher. With the use of IMVs, this diminution in possible researcher effects stems from the fact that it is the informant, and not the researcher, who controls the actual collection of the research data.

(2) In contrast to other means of data collection, informant-made videos provide visual documentation of the work of art to which the informant is responding. This makes the informant-made video recording a complete and coherent body of data. With the exception of biographical information about the informant, no other source of data is required to make sense of the recording.

(3) Informants spontaneously provide close-ups of those parts of the work to which they refer in their verbal comments. This use of image to accompany verbal commentary greatly reduces the possibility that the researcher will misinterpret an informant's statements about the work of art.

(4) Informant-made video recordings also provide visual documentation of the informant's physical interaction with a work of art. This kinesthetic information is captured automatically without any effort on the part of the informant.

(5) No other single means of data collection can provide a simultaneous record of both verbal and visual sources of information. This unique characteristic of informant-made videos provides a basis for multiple methods of data analysis-tracking, discourse analysis, study of gestures and physical relationships-using one single primary source of information.

(6) The IMV research protocol described in this paper appears to promote informants' self-reflective awareness of their own art viewing process. This and other aspects of informant-made videos suggest a number of interesting and practical educational applications in addition to research uses.

\section{Verbal Descriptions as Research Data}

Early attempts to study the process of art viewing experience addressed important epistemological problems relating to the nature of that experience and to the methodologies suitable for its study. 
The major methodological problem to be overcome ... is how to assess aesthetic experiences. Perceptions, feelings, and appreciations are internal states, not behaviors, and thus are not directly observable. What may be observed are behaviors assumed to be closely related to and reflective of internal stages and from which inferences may be made about those internal states. (Wilson, 1974, p. 60)

Wilson criticizes methods associated with quantitative research such as studies of aesthetic preference, studies of eye movement, semantic differential techniques and others as inadequate for the study of art viewing experience because "each obscures more of the aesthetic experience than it reveals" (p.60). Wilson privileges language as the most appropriate modality from which the nature of art viewing experience can be inferred.

What is needed is a behavior which is essentially isomorphic to each of the multiple facets of an unfolding aesthetic experience.

Language-verbal descriptions-is the behavior which seems to approximate most closely the aesthetic experience. (p. 60)

Language has become widely accepted as the best source of data for the study of art viewing experience and is now used by most researchers interested in museum research (e.g., Housen, 1983; Parsons, 1987; Horner, 1988, Csikszentmihalyi \& Robinson, 1990; Weltzl-Fairchild, 1992; Dufresne-Tassé \& Lefebvre, 1995). Data collection by audio-taped interviews has become the standard procedure for this kind of research.

\section{Informant-Made Videos as Research Data}

Informant-made videotape recordings provide documentation of informants' verbal statements about their art viewing experiences and, therefore, they continue the tradition of using language as a primary source of data for the study of such experiences. In addition, these videos produce a visual record of the subject's experiences from the subject's point of view; thus, they provide sources of information not available to the researcher using audio-recording technology.

As a research tool, informant-made video is rooted in the practice of

2In 1985, Felix-Louis Regnault (assisted by Charles Comte) filmed the pottery fabrication process used by a WestAfrican woman. ethnographic filmmaking. Anthropologists and ethnographers have used filmmaking in the course of their study of tribal societies and ethnic groups since the late 19th century (de Brigard, 1979). 2 However, it is only recently that researchers Worth \& Adair (1972) and Bellman \& Jules-Rosette (1977) have pioneered attempts to capture on film the particular point of view of their research subjects by asking them to make films about their own cultures.

According to the ethnographer P. Loizos (1993), documentary films or videos "cannot be simple, unmediated carbon-copies of real-world events." In defending his point of view, Loizos cites the prominent film theorist Nichols who argues that documentaries retain "an indexical 
stickiness' - they represent things which happened in front of the camera, and within range of the microphone. These sound and images are representations, second-order realities, not the original, first-order happenings" (Loizos, 1993, pp. 6-7, emphasis added).

It is important to acknowledge the fact that informant-made video tapes are documentaries that provide mediated access to subjects' art viewing experience. They are the "second-order realities" that Nichols describes above. Likewise, the camera is not meant as a substitute for the human eye, but rather as a tool by which subjects will communicate, to the best of their abilities, their response to specific works of art. The extent of informants' skills in using the "palmcorder" has an impact as well on the tapes they will produce. Moreover, the success of verbal interviews with informants also depends on subjects' skills in communicating their experience and, lest we forget, informants' verbal accounts also provide only mediated access to informants' internal experiences. In this sense, audiotaped interviews are also "second-order realities." It is only the art viewing experience itself that can qualify as "the original, firstorder happening." In the case of verbal interviewing, it is the technology of language itself, vocabulary and syntax, that interposes itself between the informant's experience and his or her ability to formulate an account of it. There is no such thing as direct, non-mediated, access to the realm of individuals' subjective experiences, no matter whether these experiences are related or not to art viewing experiences.

In sum, a methodology based on informant-made videos is probably just as suited to the study of art viewing experience as more traditional methods. In an attempt to identify the strengths and weaknesses of this new approach, this study set out to test and validate the use of informantmade videos as a form of research data.

\section{A Study of a Methodology Based on Informant-Made Videos}

\section{Method}

Informants. Ten adult informants were recruited for participation in this study. The selection of participants was guided by one criteria only: whether or not participants could be classified as either visual arts experts or non-experts. Experts were defined as those informants who had professional university training in the visual arts and/or were involved in careers where such training (or a recognized equivalent) was an entry-level requirement. In turn, non-experts were defined as participants with university training in any discipline except the fine arts. Altogether, five expert and five non-expert informants were recruited for participation in the study. The overall make-up of the group also consisted of equal num- 
3This study was conducted in Canada's national capital, (Ottawa,

Ontario) which is populated almost equally by Anglophones and Francophones.
4Each informant was read the following instructions. "First, take 5 minutes to explore the work of art without using the camera in order to come to some kind of appreciation of it. Second, in your video try to communicate to me your understanding of the work of art as well as the various ideas, steps, intuitions, and feelings that led you to it. Give your response to the work by retracing chronologically the steps by which you became acquainted with the work: Where did you begin? What was your first reaction? What did you think about? How did your impressions change? Consider how your comments may help the viewer [of your video] to better understand your experience. Finally, your tape should be between 5 to 10 minutes in duration." bers of men and women, as well as equal numbers of francophones and anglophones. 3

Procedures. The procedure for collecting the data required for this study was the same for each and every participant. It involved four main components: 1) an audiotaped interview with each informant documenting their verbal responses to two works of art; 2) individual training for each participant on the use of the video equipment; 3 ) production by the informant of a video about two additional art viewing experiences; and 4) a follow-up interview in which both the informant and the researcher viewed and discussed the informant's own video tape.

Art Criticism Process. Informants were asked to select unfamiliar works of art for which they felt an attraction. Once a selection was made, the informants were required to view the work for five minutes before producing their videotaped response. For their informant-made videos, participants were asked to retrace chronologically the steps by which they became acquainted with the art object, thus re-creating the process used to view, contemplate, and understand that particular work of art. In doing so, participants were asked to communicate their conception of the work of art, as well as the various feelings, ideas, intuitions, insights and activities that led to this understanding. 4

The art criticism process proposed to the informants was not intended to be the re-creation of a museum visit. During a typical museum visit, viewers spend very little time looking at each work of art, and they often begin verbalizing an opinion and discussing the art object with others after only a few seconds of looking. The art criticism process used in this research was designed to study the informants' full potential for responding to works of art. By separating the art criticism process into two distinct activities-looking and verbalizing-we hoped to encourage our informants to look carefully at the work of art in order to see it and understand it better. The depth and breadth of informants' videotaped responses attest to the fact that, indeed, this did happen. By studying the characteristics that determine informants' true potential for understanding works of art, we hope to eventually propose practical strategies for encouraging such responses from museum visitors.

Field Site. Research activities were conducted at the National Gallery of Canada (Ottawa, Ontario). For their participation in the project, informants chose works of art from exhibitions of the Gallery's permanent collections.

Equipment. All informants used the same camcorder-an $8 \mathrm{~mm}$ Sony CCD-TR51-for the production of all of their videotapes. This lightweight (590 grams) "palmcorder" is equipped with a $6 \mathrm{x}$ power zoom, a TTL autofocus system, a monochrome viewfinder and an incorporated 
omni-directional stereo microphone. The camera was powered using lithium battery packs, which provided 55 minutes of operating time.

Treatment of Data. The research protocol yielded a data set for each informant which comprised a 10 - to 15 -minute audio-taped recording of a baseline response to two works of art, a 10- to 15-minute informantmade video recording about two additional works of art, a transcript of a follow-up interview and, finally, the informant's biographical profile.

First, the informant-made videos were analyzed for cinematographic orientation. Second, the non-narrated segments of the videos were carefully scrutinized. Third, discourse analysis was conducted using the verbal transcripts of the tapes. In all cases, analysis was undertaken in such a way as to allow for a comparison of results between the five expert and the five non-expert informants participating in the study. Comparisons were also made between data obtained by analyzing the informant-made video tapes with data obtained using the more traditional audio-tape recording as the data collection method.

\section{Examples of the Analysis of Informant-Made Videos}

The various analyses 5 conducted in the course of this research project are presented here as examples of some of the many ways in which videographic data can be scrutinized. It is important to note that analyses can be conducted on the visual data, as well as the verbal data contained in the informant-made videos. Furthermore, analyses can focus on technical, structural, and content-related aspects of the videos, as well as on the inter-related nature of these different kinds of data. The objectives of a research project should be the determining factor in deciding what kinds of data analyses are most appropriate in specific circumstances.

\section{Cinematographic Orientation of the Videos}

Analysis of the cinematographic orientation of informant-made videos can be conducted in order to determine the nature of the intellectual stance taken by the informants during the production of their video tapes. For example, did the informants intend their videos to be objective descriptions, critical reviews, or personal interpretations of the work of art?

For the purposes of this study, Terry Barrett's (1986) Typology for the Interpretation of Photographs 6 was selected as an instrument by which to conduct the analysis. Two judges determined that all ten informant-made videos met Barrett's criteria for classification as interpretations. Barrett defines interpretations as

nonfalsifiable explanations which are analogous to metaphysical claims in language in that their makers use them to make assertions about the world independently of empirically verifiable evidence....

SPublication restrictions do not permit a detailed presentation and discussion of the results of the demonstration study. Readers are encouraged to consult the dissertation for additional information.

6Barrett's typology was deemed suitable for our purposes, even though it was originally intended for the classification of photographs, not films or videos. We had no trouble finding clear examples of films to illustrate each of the categories in the typology and, therefore, we felt that it could be used for the classification of videos without posing a risk to the validity of the instrument. This typology consists of six categories for classifying photographs: description, explanation, interpretation, ethical evaluation, aesthetic evaluation and theorization (Barrett, 1986, p.55) 
Interpretive photographs depict an intentionally subjective understanding of phenomena and generally point up the world-views of the photographers who made them" (pp. 56-57).

In this study, the consistency in the cinematographic orientation of the videos is an indication that the expert and non-expert informants actually proceeded in a similar fashion when looking at and responding to the works of art. Further support for this conclusion is provided by the results of the discourse analysis-reported elsewhere in this article-which found few differences in the expert and non-expert informants' use of psychological operations as manifested in the verbal descriptions of their encounters with the works of art. However, further analysis revealed differences in the kinds of connections that the informants established between themselves and the works of art. Non-expert informants tended to relate the works of art to personal concerns, such as relationships, trav$\mathrm{el}$, and current events, while expert informants tended to make connections between the art objects and their knowledge of art history and art theory.

\section{Analysis of Non-narrated Video Segments}

The audio-visual idiom of film and video supports the encoding of information in visual as well as verbal forms. For this reason, the non-narrated segments of informant-made videos sometimes convey information that is of considerable interest to researchers.

Approximately one-tenth of the total duration of the informant-made videos in this demonstration study consisted of non-narrated segments. Three categories were used in order to classify these segments. The categories describe the informant's intent in producing non-narrated segments: 1) To show or contemplate the work of art thus allowing it to "speak" for itself; 2) To reflect while in the process of commenting on the work of art; 3) To make a technical adjustment to the operation of the video camera or to the video image.

A noticeable difference between the two groups in this study was apparent in that the experts devoted $74.5 \%$ of all non-narrated segments to the purpose of showing the work of art (Category 1), while the nonexperts dedicated a somewhat smaller proportion (61.5\%) of their total non-narrated segments to the same end. However, within the expert group, two informants together accounted for most of the variance that makes up this difference. Of the non-expert participants, one informant accounted for most of the variance in this category within his own group. It was found that these three informants had one thing in common: they all had a considerable amount of video training and experience. These results indicate that the determining factor in the informants' use of this category of non-narrated segments was related to their professional train- 
ing in video production. It appears that it is during this training that the informants in question acquired notions about the communicative potential of non-narrated video images.

Examining informants' use of the first category of non-narrated segments offers an opportunity for considering some of the assumptions that informants may have about the nature of the art viewing experience. The inclusion of non-narrated segments, in which the work of art is simply presented visually without any of the interpretative and contextual clues normally provided by the narrator's commentary, suggests that sometimes informants assume that other viewers will see the work of art in exactly the same way as they do. They may believe that certain aspects of the work of art are obvious to everyone; otherwise, they would feel compelled to comment on the image in question in order to avoid a misunderstanding. In fact, by using non-narrated segments in their videos, they are inviting others to share in a common experience of looking at the works of art which, from an epistemological point of view, is impossible. In using these segments, informants stop communicating their own experience and, instead, begin to unintentionally encourage others-those in their intended audience-to embark on an art viewing experience of their own.

Furthermore, in making use of non-narrated segments, informants are providing their audience with opportunities to look at the work of art in question. In doing so, they may be assuming that looking is the same as seeing, and thatseeing is the equivalent of understanding. The following citation explains why such suppositions about the nature of the art viewing experience are problematic:

Art is invisible in two important ways-what awaits and what hides. For the first, much of the art in the art awaits finding. It simply is not seen at first. To get the most out of the work, you need to find it. For the second, the artist's strategies are hidden, like backstage machinery. If you find that sort of thing interesting, you may want to search out what hides as well as what awaits. Moreover, discovering what hides may feed back to disclose more of what awaits, which you missed when you sought it directly. (Perkins, 1994, p.

\section{Discourse Analysis}

As part of the production of their informant-made videos, study participants provide verbal descriptions of their experiences, which are recorded by the camcorder's built-in microphone. These comments are a valuable source of information, and they can be the focus of a number of different types of analyses.

In this study, discourse analysis was used for a detailed examination of the verbal transcripts of the soundtracks of selected informant-made 
Richard Lachapelle

videos. The objective of this discourse analysis was to study the course of informants' art criticism activities by identifying the various knowledgeseeking strategies used by the informants in their responses to the works of art. This analysis was conducted using an instrument developed by Colette Dufresne-Tassé, Thérèse Lapointe, Carole Morelli and Estelle Chamberland (1991) for the purpose of studying the experiences of adult museum visitors. Through a thorough examination of museum visitors' verbalizations, the instrument is able to identify various aspects of their psychological experience. These aspects include the mental operations used by the visitor while viewing and talking about a museum object, as well as the psychological domains from which these operations originate: cognition, affect, or imagination.

Use of the instrument consists of assigning identified units of speech, taken from the informant's statements about a specific object, to one of twelve independent and mutually exclusive categories. 7 These categories

${ }^{7}$ Two judges coded all of the transcripts used in the discourse analysis. Inter-rater reliability was high: initial disagreement between the two sets of ratings for each transcript varied from 10 to $20 \%$. The judges were eventually able to agree on ratings for all of the units of speech except for one: this unit was rejected as being too ambiguous to code. represent the thought processes that visitors use while viewing the object. In this instrument, these thought processes are referred to as "operations". The definition of the term operation used by Dufresne-Tassé et al. (1991, p. 285, as quoted from Piétron, 1963) is taken from Piaget: an operation is deemed to be "a reversible internalized action that is coordinated with others according to an overall structure" [author's translation]. The twelve operations are: to manifest, to note or state, to identify, to recall, to associate, to compare, to comprehend, to justify or explain, to resolve, modify or suggest, to situate oneself, to verify, and to evaluate.

In addition to the coding of operations by category, another feature of the instrument permits the coding of each operation according to three different domains of experience: affect, cognition, or imagination. Experience has an affective structure when "the museum visitor is dealing with his [or her] internal states, his [or her] emotional reactions, when he [or she] attributes to something or someone a qualifier that denotes attraction, pleasure, repulsion or displeasure" (Dufresne-Tassé \& Lefebvre, 1995, p. 49). There is a cognitive structure to the visitor's experience when he or she "deals with an element as though it was a fact, a data, a rule or a principle" (1993, p. 49). There is an imaginary structure to the visitor's experience when he or she formulates "a personal construct resulting out of his [or her] immediate experience, or when he [or she] evokes, as Kearney (1988) described it, a past, present or future reality that is not present in the museum itself' (Dufresne-Tassé \& Lefebvre, 1995, p. 49) [author's translation].

No noticeable differences were found in the expert and non-expert informants' use of mental operations during the process of art viewing. However, discourse analysis did reveal that non-experts favored the use of a cognitive approach during their attempts at understanding the works of 
art, whereas experts tended to use both cognition and imagination almost equally. Furthermore, expert informants formulated a greater number of hypotheses about the meaning of the works of art.

The expert informants' greater use of imagination is probably related to their professional training in the arts: such training encourages and reinforces a creative approach to activities like art production and art criticism. Furthermore, expert informants' tendency to formulate a greater number of hypotheses about the meaning of the works of art is related, no doubt, to their greater use of the domain of imagination, since the formulation of hypotheses requires the use of imagination.

\section{Comparison of the Two Data Collection Methods}

In this study, discourse analysis was also used to compare the two different data collection methods featured in the research project: the audiotaped interviews and the informant-made videos. Given the importance of language as a source of data for the study of art viewing experience (see the section entitled "Verbal Descriptions as Research Data"), this comparison was undertaken in order to determine if IMVs can provide verbal data of the same quality as audiotaped interviews. In this regard, differences between the two sets of data were found, but most of these differences are attributable to the fact that the analysis consisted in a comparison of the same informant's responses to two different works of art. However, for three of the four informants (whose tapes were selected at random for this analysis) a noticeable reduction in the use of the operation "To State" was observed in the videotaped accounts. With this one exception, the verbal data collected using informant-made videos compared quite favorably to the verbal data collected using audiotape recordings.

During follow-up interviews (conducted at the end of each participant's session), informants were asked to comment on the strengths and weaknesses of the two data collection methods used in the study: the audiotaped interviews and the informant-made videos. All informants felt that it was possible to effectively communicate their ideas about the works of art using the camcorder. Two informants felt that each technology presented certain advantages and that, in choosing one method over the other, researchers should be guided by the needs of their research. Three informants reported that producing informant-made videos eliminated the need to describe the work of art while responding to it verbally. This explains the reduction in the use of the operation "To State" in the informant-made videos. Finally, three informants felt that the video data was somehow more complete because it provided an image of the work of art to which they were responding (Lachapelle, 1994, pp. 237-239). 


\section{Discussion}

The examples of the methods of analysis provided in the previous section of the paper highlight some of ways in which the data from informantmade videos can be useful to researchers. In this part of the paper, the potential of other features of the new research tool are presented and discussed based on observations made during the course of the present study.

\section{The Meaning of Filmmaking Techniques}

The informant-made videos completed during this research project demonstrate that filmmaking techniques can convey certain information about the videographer's interaction with the work of art. The meaning of the use of some techniques can change depending on the context in which the technique is employed. In each case, the narration of a particular segment, as well as the particularities of the segments that precede or follow it, provide the additional information necessary for deciphering the exact meaning of the particular use of a technique. For example, some zoom-ins are clearly meant to be interpreted as the equivalent of a gesture, as if the informant was pointing out a part of the painting with his or her arm, hand and fingers, and saying: "Here, this is what I want you to look at." At other times, a zoom-in can mean something entirely different: a zoom-in followed by a pan over the surface of the painting may represent an attempt by the informant to search for and relocate some lost detail, as if to say: "Where is that strange mark on the surface of the canvas? I can't find it anymore." Again, it is the contextual clues provided within the video tape that allow the researcher to discern the specific meaning of a particular use of a filming technique.

\section{A Source of Kinesthetic Information}

An interesting characteristic of informant-made videos is their ability to capture kinesthetic information about the way viewers negotiate the physical space surrounding a two or three-dimensional work of art and, in the case of sculptural installations, the space within it. Informants document this information automatically, without deliberate effort, whenever they use the camcorder to record their impressions about a work of art. As a separate set of information, kinesthetic data can be used for a number of different types of studies: (a) the mapping of visitors' trajectories through exhibition galleries; (b) the evaluation of the effectiveness of exhibition design; (c) an examination of the factors that influence the way a viewer directs his or her attention to various features of a work of art.

Another advantage of informant-made videos is that it offers the possibility of following up on data collection with additional information-seeking activities. For example, the protocol used for this study included a followup interview that took place once the informant had completed his or her 
video. During the course of this interview, the informant and the researcher reviewed the informant's videotape together and, in doing so, they clarified and expanded the content of the videos. However, the follow-up interviews provided two unexpected types of information about the informants' art viewing experiences. First, in some cases, viewing their tapes led some informants to additional insights about the meaning of a work of art. Second, for a majority of informants, reviewing their videos brought them to a better understanding of their own personal art-viewing process.

New insight into the works of art. On a few occasions, viewing the videotapes did help the informants to gain new insight into the meaning of the work of art about which they had just made videos. For example, upon viewing his video, one non-expert informant came to understand why the artist, William Kurelek, decided to group the six panels of his large mural, The Ukrainian Pioneer (1971), into two distinct triptychs (with panels one, two and three together, and panels four, five and six together) with a space separating the two. The landscape depicted in the fourth panel is the exact same geographical site as that represented in the third panel, but the land has been dramatically transformed from a forest to a farmer's field. In the sequence of panels, this transformation is not depicted: instead, the space between the two triptychs is intended as a symbolic rupture representing the dramatic transformation of the land as well as the tremendous breach between the pioneers' present and former lives. As the following citation attests, Roger came to understand this only while viewing his video during the follow-up interview:

If you don't mind, please stop the tape for a few minutes....I've just now understood how he [the artist] has separated the work into two units...It's because he hasn't shown all the work required to get to that road there. It's as if there is a break....the work to be done [on the left side] and the work that is well underway [on the right side]. (Lachapelle, 1993, p. 66) (author's translation)

Increased awareness of the art viewing process. One effect of the followup interviews was quite widespread. By viewing their completed videos, most informants deepened an emerging awareness and understanding about their own art viewing practices that was, in part, a result of their participation in the study. Here is one example, from a follow-up interview, of an informant's statements about her insights into her own particular approach to viewing works of art.

I guess what I'm just realizing is how I just described the work.

I...looked at the medium, then started thinking about the content, and then I sort of used both [approaches], going back and forth (Lachapelle, 1993, p. 331) 


\section{Educational Applications of Informant-made Videos}

The video production and follow-up procedures used in this study did seem to promote a greater self-awareness about the art viewing process. This suggests there may be aspects of the methodology used here that might be useful in other contexts, such as in the teaching of art criticism and art history.

First, informant-made videos are a unique way of responding to and learning about art. The use of the video camera to record a response makes this approach a novel and interesting way to view and contemplate works of art. All of the informants who participated in this study reported and emphasized the fact that they enjoyed the activity of producing their videos.

Second, informant-made videos have the potential to be an exciting and useful substitute for a written research paper on a specific art work or art-related topic. High school students, in particular, may be more motivated to produce a video than a written paper. An approach based on the use of IMVs may help some students in overcoming difficulties related to their ability to communicate in written form. Teachers could ask students to use the camcorder to document: (a) their initial response to a work of art; (b) their attempts to locate more information about it, and (c) their revised interpretation of the art object as a result of their investigations. Furthermore, the results of these video assignments could be shared with fellow students in the form of a class presentation.

Third, teachers can also use an approach based on informant-made videos as a way of encouraging reflective practice on the part of the students in regard to their art viewing and art understanding behavior. In other words, students can use informant-made videos in order to gain insight into the strengths and weaknesses of their approach to art criticism. By producing and then carefully reviewing a videotaped response to a work of art, high school or college students can be encouraged to take a step back from the immediacy of their art viewing experience in order to examine the processes that they use in their attempts to understand works of art. By reviewing their tapes and discussing them with their teacher, learners can become aware of any recurring habits in their approach to viewing works of art. Teachers may also invite students to compare their viewing strategies with those of other students in order to better identify common strengths and weaknesses. By identifying the strategies that are useful for responding to art, students may choose to emphasize these in future encounters with works of art. However, they can also identify and then attempt to eliminate habits that hinder their learning while, at the same time, endeavor to develop new, more useful, ways of exploring the art object. 
Fourth, informant-made videos also have great potential as a teaching resource. Professional associations in art education may wish to produce a series of informant-made videos in which art experts, such as art critics or art historians, highlight the art criticism process that they use in responding to or studying historical and contemporary works of art. Artists could also participate in the production of such videos. In addition to communicating how they respond to works of art, artists could also produce IMVs about the creative processes that they use in their studio work. Teachers, at all levels of the education system, will likely find that these videos on expertise in art are useful as a tool for teaching ways in which their students can improve their art criticism and art production practices. In teacher education, such videos could be valuable aids for preparing student-teachers to teach art criticism, aesthetics, art history, and studio production.

Fifth, informant-made videos can be used for the purposes of student evaluation in much the same manner as a written paper. When using informant-made videos for teaching purposes, there is no need to resort to the same type of complicated and time-consuming analyses that are used for evaluating the results of a research project. Teachers can evaluate student videos by applying the same criteria that they use for grading written papers. Examples of possible criteria include: clarity of objectives, quality of argumentation, quality of documentation, clarity of expression, originality of ideas, and overall creativity.

Sixth, in a slightly different approach to student evaluation, informantmade videos can be used to track the progress of students involved in learning the skills of art criticism. In the same way that an art portfolio is useful in evaluating a student's progress in art production by allowing for a comparison between earlier and more recent work, a portfolio of informant-made videos can give evidence of progress in a student's ability to engage in art criticism. For example, comparisons can be made between informant-made videos produced by a student at the beginning and at the end of a school year. In most cases, by comparing assignments produced over a period of time, a teacher can easily determine whether or not a student has progressed in a specific aspect of their learning during the period of time in question. It is also a useful way for the teacher to identify weaknesses in the student's performance that will require further attention.

\section{Conclusions}

This study has demonstrated that informants were able to use a camcorder in order to effectively record and communicate their viewing experiences with specific works of art.

While the comparison of the video-taped and audiotaped sessions of four of the informants, using discourse analysis, validates the use of infor- 
mants' videos as a means of collecting verbal statements about works of art, the new research method proposed in this article has many other characteristics that make it particularly useful for meeting certain types of research objectives. Informant-made videos are ideally suited to situations where research goals make it is desirable to share or relinquish control over the data collection process in favor of the research informant; this reduces the possibility of researcher bias and increases the cogency of the information collected. In situations where the researcher must maintain a high degree of control over the process of data acquisition, audio-taped interviews remain the best option. Through his or her questions, the interviewer can exert a greater degree of direction over the data collection; however, the cost of more control is an increased risk of researcher effects on the information collected. In selecting one method over the other, researchers must be guided by the research objectives, the role of informants, and the types of analyses of their particular study; they must consider whether these are congruent with a data collection method based on audiotaped interviews or informant-made videos.

Furthermore, we have seen that informant-made videos provide types of information that may be very useful in some cases. In addition to a record of the informant's comments, IMVs supply visual documentation of the works of art used in the research. Such documentation reduces the likelihood that researchers will misinterpret an informant's statements about the art object. IMVs also provide a record of the informant's physical interaction with the work of art. Moreover, informant-made videos provide a basis for multiple and complementary methods of data analysis. Again, in selecting informant-based videos as a data collection method, researchers should be guided by their needs for these types of complementary information.

Finally, we have seen that, in addition to research applications, there are many educational uses for IMVs. Informant-made videos provide an singular approach for responding to and learning about art. In addition to the examples presented earlier, it is not difficult to imagine many other possible educational uses for this new tool. Informant-made videos can be useful in research and teaching whenever the specific point of view of the research subject or student is considered useful or desirable.

\section{References}

$\rightarrow$ Barrett, T. (1986). A theoretical construct for interpreting photographs. Studies in Art Education, 27(2), $52-60$.

Bellman, B., \& Jules-Rosette, B. (1977). A paradigm for looking: Cross-cultural research with visual media. Norwood, NJ: Ablex Publishing Corporation.

de Brigard, E. (1979). Historique du film ethnographique. In C. de France (Ed.), Pour une anthropologie visuelle (pp. 21-51). Paris: Mouton Éditeur. 
Csikszentmihalyi, M., \& Robinson, R. E. (1990). The art of seeing: An interpretation of the aesthetic encounter. Malibu, CA: The J. Paul Getty Trust.

Dufresne-Tassé, C., \& Lefebvre, A. (1995). Psychologie du visiteur de musée. Ville La Salle, QC: Éditions Hurtubise HMH Ltée.

Dufresne-Tassé, C., Lapointe, T., Morelli, C., \& Chamberland, E. (1991). L'apprentissage de l'adulte au musée et l'instrument pour l'étudier. Revue canadienne de l'éducation (16)(3), 281-291.

Heider, K. G. (1976). Ethnographic film. Austin: University of Texas Press.

Horner, S. (1988). 2C \& Not 2B; That is not a question. Unpublished manuscript, Concordia University.

Housen, A. (1983). The eye of the beholder: Measuring aesthetic development. Unpublished doctoral dissertation, Harvard Graduate School of Education, Boston, Mass.

Kurelek, W. (1971). The Ukrainian pioneer[Painting]. National Gallery of Canada, Ottawa.

Lachapelle, R. (1993). [Transcripts of informants' interviews and video tapes]. Unpublished raw data.

Lachapelle, R. (1994). Aesthetic understanding as informed experience: Ten informant-made videographic accounts about the process of aesthetic learning. Unpublished doctoral dissertation, Concordia University, Montreal, Quebec.

Loizos, P. (1993). Innovation in ethnographic film, 1955-85. In Innovation in ethnographic film: From innocence to self-consciousness, 1955-85(pp. 5-15). Chicago: The University of Chicago Press.

Parsons, M. (1987). How we understand art: A cognitive developmental account of aesthetic experience. New York: Cambridge University Press.

Perkins, D. N. (1994). The intelligent eye: Learning to think by looking at art. Santa Monica, CA: The Getty Center for Education in the Arts.

Weltzl-Fairchild, A. (1992). A study of aesthetic response: Analysis of the verbal and non-verbal responses of children to art reproductions. Unpublished doctoral dissertation, Université du Québec à Montréal, Montreal.

$\rightarrow$ Wilson, B. (1974). One view of the past and future of research in aesthetic education. Journal of Aesthetic Education, 8(3), 59-67.

Worth, S., \& Adair, J. (1972). Through Navajo eyes: An exploration in film communication and anthropology. Bloomington: Indiana University Press. 